- Case Report

\title{
latrogenic Development of Cerebrospinal Fluid Leakage in Diagnosing Spontaneous Intracranial Hypotension
}

\author{
Chang-Joon Lee, Sung-Min Shim, Sang-Hyeon Cho, Jae-Ho Park, Young Ki Kim* \\ Department of Anesthesiology and Pain Medicine, Gangneung Asan Hospital, Gangneung, Korea
}

\begin{abstract}
A 34-year-old woman came to the emergency room complaining of a severe orthostatic headache. Results of a cerebrospinal fluid tap and brain computed tomography were normal. Based on her history and symptoms, she was found to have spontaneous intracranial hypotension. She was hospitalized and her symptoms improved with conservative treatment. On the next day, her headache suddenly worsened. Cisternography was performed to confirm the diagnosis and determine the spinal level of her cerebrospinal fluid leak. It revealed multiple cerebrospinal fluid leaks in the lumbar and upper thoracic regions. It was strongly believed that she had an iatrogenic cerebrospinal fluid leak in the lumbar region. An epidural blood patch was performed level by level on the lumbar and upper thoracic regions. Her symptoms resolved after the epidural blood patch and she was later discharged without any complications. In this case, an iatrogenic cerebrospinal fluid leak was caused by a dural puncture made while diagnosing spontaneous intracranial hypotension, which is always a risk and hampers the patient's progress. Therefore, in cases of spontaneous intracranial hypotension, an effort to minimize dural punctures is needed and a non-invasive test such as magnetic resonance imaging should be considered first.
\end{abstract}

Keywords: Cerebrospinal Fluid; Epidural Blood Patch; Post-Dural Puncture Headache; Intracranial Hypotension

Received: April 14, 2017, Revised: May 23, 2017, Accepted: May 23, 2017

*Corresponding Author: Young Ki Kim https://orcid.org/0000-0002-7205-6746

Tel: +82-33-610-3409, Fax: +82-33-641-8180, E-mail: ykkim@gnah.co.kr 


\section{INTRODUCTION}

In the past, spontaneous intracranial hypotension (SIH) was considered an extremely rare disease, but recently it has been considered a routine cause of headaches. In one study, the prevalence of SIH was estimated at 1:50,000. ${ }^{1)} \mathrm{SIH}$ develops without any preceding events such as trauma, head or neck surgery, a procedure that punctured the dura, or other medical conditions such as dehydration or diabetic ketoacidosis. SIH is caused by a spontaneous cerebrospinal fluid (CSF) leak and the characteristic symptom is a postural headache. Neck stiffness, nausea, vomiting, or visual disturbance may also occur. Diagnosis is made based on the patient's history and symptoms, as well as laboratory findings. A CSF study, computed tomography (CT), magnetic resonance imaging (MRI), or radionuclide cisternography may be helpful for the differential diagnosis. In most cases, patients undergo a lumbar puncture for CSF analysis or cisternography to confirm $\mathrm{SIH}$ or to rule out other diseases. However, the lumbar puncture itself may cause secondary intracranial hypotension due to the trauma to the dura. There are no reported cases of SIH where the patient also suffered from a CSF leak due to the iatrogenic dural puncture. Here, we present the case of a young female patient who was diagnosed with SIH and was strongly believed to have a post-dural puncture CSF leak. We also discuss the relevant literature.

\section{CASE REPORT}

A 34-year-old woman presented to the emergency room (ER) complaining of a persistent severe headache. Her headache had started 4 days earlier after vomiting and she was unable to walk due to the headache. She was previously healthy and was never treated for any medical disorders or chronic headache disorders. She visited a local hospital where a brain MRI and magnetic resonance (MR) angiography were performed, but there were no abnormal findings. She felt better when she lied down. Numeric rating scale (NRS, 'no pain' is score of 0 and 'worst imaginable pain' is score of 10) was 1-2. The pain aggravated when sitting up or standing up within 15 minutes. NRS was 10. She was diagnosed with SIH and treated conservatively, but her symptoms did not improve. She had no history of surgeries, injections, nerve blocks, or recent trauma, and she was never diagnosed with a connective tissue disease (CTD). She described her headache as generally throbbing, especially in the occipital area. Her vital signs were stable and she did not have a fever. She also complained of eye pain, dizziness, and nausea.

She was referred to the neurology department and blood laboratory tests and CSF tapping were performed in the ER. A lumbar puncture was made at the L4-5 interspinous space using a midline approach with a 22 gauge Quincke needle. The puncture was made successfully on the second attempt. The opening pressure was $46 \mathrm{mmH}_{2} \mathrm{O}$ and 4 $\mathrm{mL}$ of CSF was naturally drained for analysis. There was no adverse event during the procedure and the patient was placed on bedrest for 4 hours. Afterwards, a brain CT study was done. The results of all tests were normal. Then, a neurologist assessed her SIH and she was hospitalized in the general ward for further evaluation and treatment.

From hospital day (HD) 1, she started to receive parenteral hydration. Normal saline $80 \mathrm{~mL} / \mathrm{h}$ was infused intravenously. Opioid medication was administered intravenously as needed to control her headache. Her pain decreased to a NRS of 4 and she could go to the toilet independently. However, she was still not able to return to her activities of daily living because of pain.

On HD 3, radionuclide cisternography was performed to confirm her diagnosis and assess the CSF leak. A 20 gauge Quincke needle was used to introduce the radionuclide isotope (Tc-99m DTPA 2.8mCi) into the intrathecal space. The procedure was performed successfully on the third attempt at the L3-4 interspinous space using a midline approach. Whole body cisternographic images were obtained at 30 minutes, 2 hours, 4 hours, and 6 hours after injection.

On HD 4, she complained that her orthostatic headache had worsened to NRS 9. The cisternography revealed multiple CSF leaks along the lumbar region and in the upper thoracic region (Figure 1). Early and prominent visualization of kidney and bladder activity was observed in the images and was consistent with CSF leakage. Immediately, she received an epidural blood patch (EBP) to the L4-5 level with $15 \mathrm{~mL}$ of autologous blood under fluoroscopic guidance. After the procedure, her headache improved to NRS 2. On HD 5, some aching pain still remained after the first EBP and she received another EBP to the upper thoracic region under fluoroscopic guidance. The EBP was done at the T3-4 level with $10 \mathrm{~mL}$ of her own blood. After the procedure, her pain improved gradually to NRS 1 . Her vital signs were stable, and she was discharged on HD 7 without any complications.

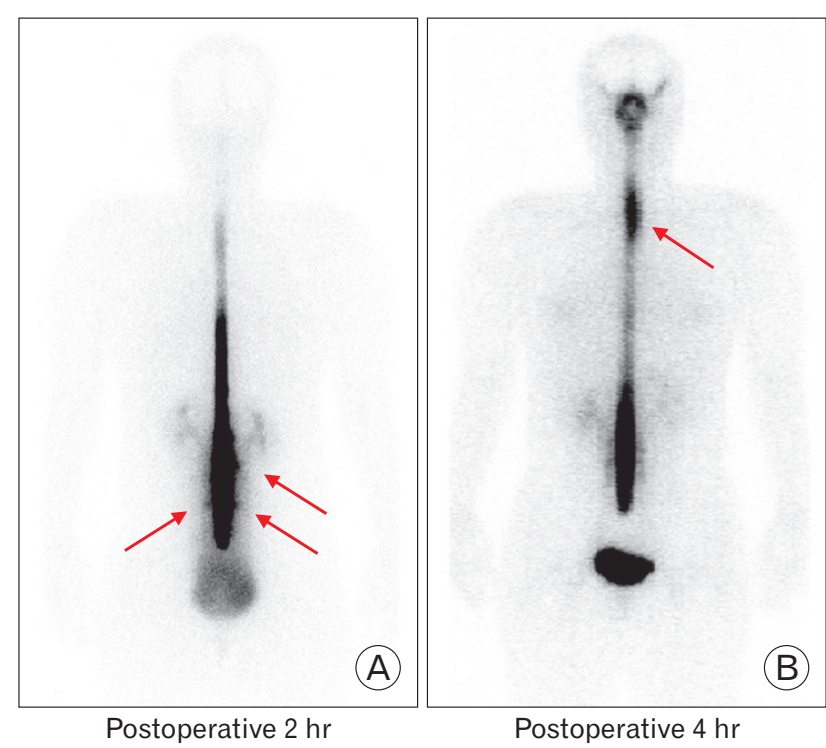

Figure 1. Citernographic images. (A) Multiple cerebrospinal fliud leaks in the lumbar regions (arrows). (B) Cerebrospinal fliud leaks in the thoracic regions (arrow). 


\section{DISCUSSION}

Intracranial hypotension syndrome (IHS) is a single pathophysiological entity of diverse origin that occurs due to a persistent CSF leak. It is divided into primary and secondary IHS according to the cause. Spontaneous or primary IHS develops without any traumatic events. Secondary IHS occurs after trauma to the dura. This trauma could include a dural puncture procedures, such as CSF tapping, spinal anesthesia, incidental dural puncture during epidural nerve block, or surgery. ${ }^{2)}$ Post-dural puncture headache (PDPH) is a clinical syndrome that commonly causes secondary IHS. IHS can be confirmed if the CSF opening pressure during the lumbar puncture is less than $60 \mathrm{mmH}_{2} \mathrm{O}^{3)}$

Orthostatic headaches that typically disappear after lying down are the characteristic symptom of IHS. IHS may also cause nausea, vomiting, visual disturbance, neck stiffness, and cranial nerve paresis. A diagnosis of IHS is based on patient history and the presence of orthostatic headaches. To evaluate IHS non-invasively, an MRI of the brain and spine may be used. The major abnormalities demonstrated on MR imaging studies in patients with SIH are diffuse thickening of the meninges with enhancement, engorged venous sinuses, subdural fluid collections, and a downward displacement of the brain. ${ }^{4)}$ Imaging also aids in differentiating IHS from other diseases. However, the costs of imaging are comparatively high compared to other diagnostic examinations. Furthermore, not all cases of IHS will be found using MRI because some cases, such as the case presented here, are associated with normal MRI findings. ${ }^{4)}$ Invasive tests, such as CSF tapping, CT myelography, and radionuclide cisternography, are preferred to confirm IHS. CSF tapping is commonly the initial test for checking the CSF opening pressure and to perform differential diagnosis with a CSF analysis. Radionuclide cisternography is the most efficient tool to confirm the diagnosis and localize the CSF leak to a spinal level. ${ }^{1)}$

Once the diagnosis is made, conservative treatment is started. Bed rest, fluid therapy, and caffeine intake may be helpful. ${ }^{1)}$ If there is no improvement with conservative management, an autologous EBP may be considered. ${ }^{5)}$ There are two mechanisms by which an EBP improves IHS. First, the procedure directly seals the CSF leakage site. Second, the injected blood raises the epidural pressure and may interfere with CSF flow and absorption and reduce leakage. ${ }^{5)}$

In this case, when the patient was suspected of having SIH, conservative management was initiated. Although her symptoms initially improved after the first lumbar puncture in ER, they worsened (NRS 4 to 9) after the lumbar puncture for the cisternography. It was suspected that her worsened headache was due to the combination of the iatrogenic post-dural puncture CSF leak and her underlying SIH. The following reasons support it.

First, her symptoms got worse suddenly on the day after the lumbar puncture for cisternography (NRS 4 to 9). Usually, PDPH is known to start within the first 48 hours of a traumatic event. Second, she had multiple risk factors for PDPH. Young, female, multiple dural punctures, large needle size (22 gauge for diagnostic tapping in ER, 20 gauge for cisternography), and Quincke needle usage are all risk factors for
PDPH. ${ }^{6)}$ In one study, $36 \%$ of patients reported a headache after a dural puncture with a 22 gauge Quincke spinal needle. ${ }^{6)}$ Third, It is known that the thoracic spine or the cervicothoracic junction is a common site of CSF leakage in most SIH patients. ${ }^{4)}$ However, in this case, multiple leak sites in the lumbar region were confirmed on cisternography. Based on these findings, we concluded that the patient had an iatrogenic CSF leak and a spontaneous leak at the same time, and that the combined leaks worsened her symptoms.

SIH occurs more frequently in women than in men and mainly occurs between $30-40$ years of age. In $16 \%-38 \%$ of SIH patients, a CTD is found and the presence of a CTD is a risk factor for SIH. ${ }^{1)}$ Interestingly, these features overlap with the risk factors of PDPH though the patient, in this case, was never diagnosed with a CTD nor had any symptoms suggesting a CTD. It is not clear how a CTD contributes to PDPH but there is a study that suggests that a CTD is a risk factor of PDPH. ${ }^{7)}$ Any procedures that punctures the dura, such as a CSF analysis, CT myelography, or radionuclide cisternography, performed on patients with SIH may cause iatrogenic CSF leakage more frequently than in the general population. However, clinicians easily neglect this risk when diagnosing SIH because the patient's symptoms may not differ after the iatrogenic CSF leak occurs, and the cisternographic image cannot determine whether the CSF leak is spontaneous or iatrogenic. Sakurai et al ${ }^{8)}$ emphasized that abnormal cisternographic findings at the lumbosacral level should be carefully interpreted when diagnosing SIH to avoid confusing the findings with the iatrogenic CSF leak from administering the isotope tracer into intrathecal space. Thus, we cautiously assumed that, among SIH patients with multiple CSF leak sites, including the lumbosacral level, some of them have an iatrogenic CSF leak.

We suggest that the routine use of invasive procedures for all suspected cases of SIH be avoided. CSF tapping is usually the first test performed but it may fail to obtain a sufficient specimen, known as a 'dry-tap', which may result in repeated punctures and raise the risk of an iatrogenic CSF leak. ${ }^{2)}$ The lumbar puncture for CT myelography or radionuclide cisternography also risks an iatrogenic CSF leak that may worsen the patient's symptoms and prognosis. In one study, an early lumbar EBP, without performing a lumbar puncture or identifying a CSF leak, was effective in $77 \%$ of patients who had an orthostatic headache. ${ }^{9)}$ There is also a report that in one patient a single lumbar EBP was effective in treating multiple CSF leak sites. ${ }^{1)}$ These reports imply that confirming the CSF leak and the level of the leak with dural puncturing procedures is not always essential for diagnosing and treating suspected SIH patients. In most cases of SIH, the success rate of an EBP is lower than in cases of PDPH where repeated EBPs are required. The reason for this difference may be the anatomical differences in the leak sites, a congenitally weak point in the dura, or the different EBP sites. ${ }^{10)}$ We recommend physicians consider an iatrogenic cause when the patient does not improve after one or two EBPs.

In conclusion, when a physician encounters a patient with the typical symptoms of SIH, a non-invasive test such as an MRI should be considered first as long as there is not any other disease that must be 
urgently ruled out. In diagnosing and treating cases of SIH, an effort to minimize dural punctures is needed especially in patients with multiple risk factors for PDPH. Unnecessary dural punctures may prolong the hospitalization period and worsen the patient's symptoms.

\section{CONFLICT OF INTEREST}

No potential conflict of interest relevant to this article was reported.

\section{REFERENCES}

1. Ko HC, Jung JK, Moon HS, Song JH, Eum SH, Cha YD. Treatment of spontaneous intracranial hypotension with multiple leakage sites of cerebrospinal fluid: a case report. Anesth Pain Med 2008;3:330-3.

2. Rando TA, Fishman RA. Spontaneous intracranial hypotension: report of two cases and review of the literature. Neurology 1992;42(3 Pt 1): 481-7.

3. Bell WE, Joynt RJ, Sahs AL. Low spinal fluid pressure syndromes. Neurology 1960;10:512-21.
4. Kim DK, Son JS, Kim DC, Lee JW, Lim HS. Spontaneous intracranial hypotension after post-dural puncture headache: a case report. Korean J Anesthesiol 2007;52:245-8.

5. Schievink WI. Spontaneous spinal cerebrospinal fluid leaks and intracranial hypotension. JAMA 2006;295:2286-96.

6. Turnbull DK, Shepherd DB. Post-dural puncture headache: pathogenesis, prevention and treatment. Br J Anaesth 2003;91:718-29.

7. Youngblood SC, Tolpin DA, LeMaire SA, Coselli JS, Lee VV, Cooper JR Jr. Complications of cerebrospinal fluid drainage after thoracic aortic surgery: a review of 504 patients over 5 years. J Thorac Cardiovasc Surg 2013;146:166-71.

8. Sakurai K, Nishio M, Sasaki S, Ogino H, Tohyama J, Yamada K, et al. Postpuncture CSF leakage: a potential pitfall of radionuclide cisternography. Neurology 2010;75:1730-4.

9. Berroir S, Loisel B, Ducros A, Boukobza M, Tzourio C, Valade D, et al. Early epidural blood patch in spontaneous intracranial hypotension. Neurology 2004;63:1950-1.

10. Lee JI, Roh JH, Yoon DM, Lee YW. Spontaneous intracranial hypotension and epidural blood patch: a case report. Korean J Anesthesiol 2005;48:216-9. 